University of California, Hastings College of the Law UC Hastings Scholarship Repository

Faculty Scholarship

2004

\title{
Using Mediation Techniques to Manage Conflict and Create Healthy Work Environments
}

Debra Gerardi

UC Hastings College of the Law, gerardig@uchastings.edu

Follow this and additional works at: https://repository.uchastings.edu/faculty_scholarship

\section{Recommended Citation}

Debra Gerardi, Using Mediation Techniques to Manage Conflict and Create Healthy Work Environments, 15 AACN Advanced Critical Care 182 (2004).

Available at: https://repository.uchastings.edu/faculty_scholarship/1613

This Article is brought to you for free and open access by UC Hastings Scholarship Repository. It has been accepted for inclusion in Faculty Scholarship by an authorized administrator of UC Hastings Scholarship Repository. 


\section{Using Mediation Techniques to Manage Conflict and Create Healthy Work Environments}

Debra Gerardi, RN, MPH, JD

Healthcare organizations must find ways for managing conflict and developing effective working relationships to create healthy work environments. The effects of unresolved conflict on clinical outcomes, staff retention, and the financial health of the organization lead to many unnecessary costs that divert resources from clinical care. The complexity of delivering critical care services makes conflict resolution difficult. Developing collaborative working relationships helps to manage conflict in complex environments. Working relationships are based on the ability to deal with differences. Dealing with differences requires skill development and techniques for balancing interests and communicating effectively. Techniques used by mediators are effective for resolving disputes and developing working relationships. With practice, these techniques are easily transferable to the clinical setting. Listening for understanding, reframing, elevating the definition of the problem, and forming clear agreements can foster working relationships, decrease the level of conflict, and create healthy work environments that benefit patients and professionals. (KEYWORDS: conflict, healthy work environments, mediation, working relationships)
As care of critically ill patients has advanced over the past few decades, there has been a tremendous evolution in the development of technology, advances in less invasive therapies, miraculous drugs for fighting infections and enabling organ transplantation, and an increased knowledge of physiology and genetics. The growth in the scientific aspects of care has enabled patients to receive treatment with fewer complications and better outcomes. The dramatic advances have changed the system of care delivery and will continue to expand the options for critically ill and injured patients. Unfortunately, healthcare organizations have not evolved as quickly as clinical advances, and the environment of care has evolved into a difficult and complex setting filled with poor communication, unclear policies, role confusion, turf battles, and stressful interpersonal conflicts. ${ }^{1}$

Creating a healthy work environment is as important, and perhaps more important, as mastering the newest technology. The challenge of how to develop solid working relationships in a complex and hectic clinical setting is the next step in the evolution of healthcare delivery. To address this challenge, it is beneficial to understand the costs

From Health Care Mediations, Inc, Mountain View, Ca.

Reprint requests to: Debra Gerardi, RN, MPH, JD, President and CEO, Health Care Mediations, Inc, 360 Franklin St, Mountain View, CA 94041 (dgerardi@ healthcaremediations.com). 
of conflict, the effects of organizational complexity, the barriers to managing conflict, and techniques for effective resolution of disputes. Integrating collaborative conflict management strategies into the daily activities of clinical care can improve patient outcomes, improve retention of nursing staff, and create an environment that optimizes scientific advances through enhancement of effective working relationships. ${ }^{2}$ Incorporating the skills and techniques used by mediators can facilitate collaborative working relationships and create healthy work environments. This article provides an overview of factors impacting management of clinical conflicts and an introduction to techniques used by mediators to resolve conflicts, foster collaboration, and prevent dispute escalation.

\section{$\square$ Costs and Consequences of Conflict}

The consequences of unresolved conflict are serious and greatly impact retention of qualified staff, clinical outcomes of patients, the satisfaction of patients and their families, and an organization's financial success. ${ }^{2-4}$ The direct and indirect costs of ongoing conflict organizationally include: diversion of resources to litigation, workman's compensation and disability claims; employee grievances, increased turnover, decreased morale, clinical errors, diversion of management time, unwelcome publicity, and disruption of professional relationships. ${ }^{5-7}$ Table 1 outlines several of the direct and indirect costs associated with organizational conflict.

Patients and their families are often aware of the level of teamwork and collaboration within a unit and are dependent upon the ability of the nursing staff to respond to their concerns. ${ }^{4}$ Energy that is put into managing a dysfunctional environment is energy that is unavailable for addressing the concerns and needs of patients and families. ${ }^{5}$ Clinically, outcomes are directly affected by the extent of collaboration and coordination of care. ${ }^{8-10}$ Coordinating care of critically ill patients requires clear communication and effective teamwork. The culture of the unit impacts the effectiveness of the team. Units in which staff members accept poor communication as status quo expose patients to errors and bad outcomes. ${ }^{11}$ A negative culture that perpetuates a "blame and shame" response to adverse events further contributes to an unsafe care environment. ${ }^{12}$ Additionally, once a negative culture develops, recruitment and retention of qualified nurses becomes difficult.1,2 Loss of experienced staff contributes to the stress and unit dysfunction.

\section{Barriers to Managing Conflict}

Due to the unique culture of healthcare delivery, there are many barriers that prevent effective resolution of disputes. These barriers include: time constraints, inadequate access to information, poor communication structures, unclear roles, conflicting policies, diversity of education/experience of clinicians, power imbalances, practice variations, high stakes, emotionally charged situations, and fatigue. ${ }^{13-16}$ Additionally, competing clinical and operational priorities prevent consistent focus on the management and prevention of conflict situations. ${ }^{15}$ For many nurses and physicians, there is little training or skill development in negotiation, listening, communication, or conflict resolution. ${ }^{13}$, 14 With limited training and with little priority placed on the importance of developing collaborative working relationships, there are few mentors or role models for modeling effective skills and techniques. ${ }^{13,14}$ There may be an expectation of collaboration and teamwork, but there is rarely a clear definition of collaboration, and perceptions vary across clinical disciplines. 2,17,18 Feedback within hierarchical organizations as to appropriate communication and professional interactions is skewed. ${ }^{19}$ Additionally, nurses have a tendency to avoid addressing conflict directly. ${ }^{20}$ With organizational barriers, skill-based deficiencies, few role-models, inconsistent feedback, and avoidant behaviors, it is little wonder that conflict has become an integral part of the clinical setting. The goal for successful collaboration is to overcome these barriers and stay engaged in resolving the issues.

\section{Organizational Complexity}

Assessing the complexity of the clinical environment can help to focus the way in which 
TABLE 1 - Costs of Unmanaged Conflict2-9,19

\section{Direct Costs of Conflict}

Litigation costs: attorney's fees, expert testimony, depositions, lost work time, transcripts, document production, etc.

Management productivity: time spent working on resolving conflicts rather than other managerial duties

Turnover costs for training new staff, recruitment costs

Disability/stress claims, workman's compensation claims

Regulatory fines for noncompliance or loss of contracts or provider status with insurers and Medicare/Medicaid

Costs associated with increased care expenditures for patients with preventable poor or adverse outcomes
Indirect Costs of Conflict

Team morale, motivation for organizational change, workplace relationships damaged, unresolved tensions that lead to future conflicts

Lost opportunities for pursuing capital purchases, expanding services, enhancing customer satisfaction programs, developing staff/ leaders

Costs to patients due to delays in traditional litigation can lead to more costly outlays for healthcare than if conflicts are addressed early and directly

Cost to reputation of organization and of care professional; negative publicity/ media coverage

Loss of strategic market positioning due to public disclosure of information regarding the dispute/bad public relations

Increased incidence of disruptive behavior by staff and medical professionals

Emotional costs: pain, turmoil for those involved in the conflict conflict is managed. Throughout each day, patient care relies on a complex series of interactions to coordinate scheduling, resources, information, and people. Within complex systems, it is common for patterns to form. What may appear from the outside to be chaotic, is actually a patterned network of loose linkages between multiple components of the system. ${ }^{21}$ These patterns are familiar to those who work within these complex environments.

The basic routine in an intensive care unit (ICU) is quite similar from hospital to hospital. There are parallels in how clinical orders are written and transcribed, how medications are obtained, how treatments are scheduled, and how procedures are conducted. An experienced traveling nurse or float nurse can learn what he or she needs to know to care for patients with just a few minutes of orientation as to location of medications, use of phones, and access to physicians and supplies. Additionally, communi- cation flow and behavioral norms exhibit patterns that become familiar over time and constitute the culture of the organization..$^{22}$ These patterns are learned through experience, observation, and sharing of information from person to person. Most nurses can navigate the clinical environment by asking questions and learning from others. The reliance on information sharing and relationships with coworkers allows clinicians to make sense of the environment and do the work of caring for patients. Making sense of a chaotic environment is a basic skill for those working within complex systems. ${ }^{21}$ Fostering collaborative relationships is the key to ensuring success for professionals working within complex environments and serves as a foundation for dealing with differences when they arise. ${ }^{21,23}$

The complexity of delivering critical care makes conflict management difficult and often the complexity prevents resolution of underlying causes of conflict. ${ }^{24}$ Quick fixes 
have become a common mode of problem solving as staff and managers feel overwhelmed by what it takes to achieve true issue resolution. Frequently, problems arise that become a tangled web of related issues that make it routinely difficult to develop sound comprehensive solutions that can be implemented throughout the unit or organization. For example, examining the repeated difficulty of obtaining a particular medication can result in discovery of related issues such as a shortage of pharmacy technicians, Drug Enforcement Agency or other regulatory restrictions, policy changes, vendor back-orders, or outdated medication profiles. These issues are not easily addressed and with typical management structures are definitely not within the realm of control of the staff nurse or staff pharmacist who is attempting to collaborate to meet the patients' needs. This cascade of complex problems can result in poor working relationships between the nursing unit and the pharmacy, which can lead to unresolved conflicts that resurface repeatedly and cause burn-out, frustration, apathy, and disengagement. ${ }^{25}$ The continued exposure to systemic conflict taxes the emotional resources of staff and contributes to the development of toxic environments. ${ }^{19}$ Over time, it becomes easier to walk away or to stop trying to resolve the constant stream of difficulties than invest energy in events that distract from patient care. This disengagement can fuel further frustration, decreased morale, loss of trust in the organization, and a lesser tendency toward giving colleagues the benefit of the doubt when difficulties arise. 19,20

\section{$\square$ Interdependencies and Working Relationships}

Complex systems have distinct interdependencies that enable coordination of resources and information. These interdependencies exist among departments, professional groups, administrators, and clinicians. ${ }^{21}$ Nursing relies on the pharmacy to procure medications for the unit on time. Pharmacy relies on the physicians to write the correct orders for medications. Unit staff depend on management to secure resources and provide information. Patients depend on practitioners, clin- icians depend upon support personnel, and the list goes on. These interdependencies make it essential that there be strong working relationships for care to be delivered within these complex systems.

The presence of poor working relationships leads to operational and social hassles that impact the staff's ability to provide quality care and affects recruitment and retention of nursing staff. ${ }^{25}$ Adequacy of qualified staff directly impacts patient safety. 5 Additionally, the inherent stress associated with delivering care to critically ill patients can foster inappropriate disruptive behavior that further affects retention, morale, and quality of care. ${ }^{26}$ Understanding the components of effective working relationships and intentionally integrating techniques for improving collaboration helps to manage conflict by developing the capacity to deal with differences.

As part of the Harvard Negotiation Project, Fisher and Brown ${ }^{24}$ conducted seminal research on the qualities that ensure a good working relationship. The researchers defined a working relationship as having the ability to deal with differences. Having a good working relationship depends upon balancing reason and emotion, understanding the other's needs and interests, fostering good communication, being reliable, using persuasion rather than coercion, and mutual acceptance. ${ }^{24}$ These collaborative relationships can serve as the foundation for responding to more difficult conflicts and create an atmosphere where common differences are prevented from escalating into protracted disputes. Mediators are frequently called upon to assist with conflict situations and help establish functional working relationships. Developing skills in each of these six areas is important for improving clinical negotiations and in managing conflict situations. Providing staff training is one method for developing collaborative skills. Skill development can occur through formalized training programs as well as through modeling of mediator techniques in daily clinical practice. Incorporating the techniques used by mediators is an effective way to teach collaborative skills and create functional working relationships. 


\section{What Is Mediation?}

Mediation is a conflict resolution process in which a neutral person facilitates communication, the development of understanding, and the generation of options for creative dispute resolution. ${ }^{27}$ Unlike a judge or jury, the mediator does not decide the outcome of a dispute. A mediator's role is to help participants surface issues, to create a safe space to discuss issues that may be emotionally or psychologically difficult, and to foster agreement as participants seek options that could move them forward toward workable solutions. Mediation is a useful process to use when the goal of preserving the working relationship is as important as resolving the substantive problems. Unlike litigation or arbitration, mediation allows the participants in the dispute to remain in control of the process and to contribute to the outcomes. ${ }^{27}$ Through facilitated dialogue, the participants are able to identify what is important to them and what they need to reach a solution. Mediation is voluntary and relies on the good faith participation of the people involved in the process. One of the fundamental tenets of mediation is confidentiality. To encourage honest and open communication among participants, all conversations associated with the mediation process remain confidential. As a process, mediation can be used within and external to the clinical setting and requires the use of a trained "neutral" or mediator. With sufficient training and practice, anyone can serve as a mediator provided they have no stake in the outcome of the dispute.

In the critical care setting, many conflicts arise in the normal course of care delivery. Common examples include conflicts related to end-of-life decision-making, differences regarding plan of care, scheduling and assignment making, visiting hours, access to equipment and supplies, and placement of patients. More serious disputes can arise when a medical error or adverse outcome occurs or when a patient's family is in conflict with the clinical team. Often these conflicts are managed at a cursory level, addressing obvious or expressed needs but frequently leaving underlying causes of the conflict unaddressed.23,27

It is uncommon for people to clearly identify what is driving their discomfort, whether it be a need for control, fear, perceptions of mistreatment, or allegations of injustice or unfairness. ${ }^{27}$ In a busy critical care unit, it is particularly unlikely that someone will identify their emotional or psychological needs. Generally, healthcare organizations value being in control; asking to have your emotional needs met can foster fears of appearing weak. 28 The inability to express needs directly can also be seen with patient's family members. The following is an example observed in an intensive care unit:

Patient's Husband: What is the hematocrit level? Has it come back yet? Why is her blood pressure so low? It was higher when I was in here before. Will I be able to come back in to see her if I leave to get something to eat? Last time I left, I wasn't able to get back in for 2 hours. Have you taken care of her before? She had a really good nurse yesterday. When will the doctor be here for rounds? Has he seen her scans yet? Do you know when she will be scheduled for her procedure?

\section{Observed Needs: Information}

Possible Unspoken Psychological

Needs: Control, reassurance, to appear responsible.

Possible Unspoken Emotional Needs: Fear, worry, frustration, guilt.

Addressing the husband's need for information meets one of his needs. Failure to assess and address the unspoken needs can lead to a continued barrage of questions that can result in frustration by both the nurse and the family member. Failure to acknowledge them does not lessen unspoken needs or make them disappear. Continuing to discount or ignore them can lead to stressful interactions that may result in a conflict situation.

All too often, when faced with frequent or recurring demands for information, the nurse or physician will repeatedly provide further explanation of clinical physiology, or reiterate unit policies that only serve to cause further anxiety for the family member or patient whose underlying fears remain unacknowledged. Table 2 provides examples of "symptoms" of conflict and common underlying needs and interests. Accurate assessment and avoidance of premature assumptions (Table 2) can facilitate resolution of clinical conflicts. Mediation techniques are very effective at surfacing underlying 


\section{TABLE 2 - Assessing the Conflict*}

\section{Some "Symptoms" of Conflict}

- Negative body language: eye-rolling, turning away, closed postures, facial expressions showing anger or hurt

- Repetition of story or requests

- Disruptive behavior, angry outbursts, aggressive actions (throwing objects, slamming doors)

- Avoidance behaviors: not following through, showing up late, frequent sick calls, indirect or no communication

- Sabotage, intentional misinformation, harmful gossip

- Turnover, compensation claims, disability

- Apathy, disengagement, hopelessness

Possible Underlying Needs and Interests

Driving the Conflict

- Resource needs not met: inadequate, time, staff, space, information, supplies, equipment, money

- Psychological needs not met: control, respect, authority, recognition as expert, power, safety, inclusion, sense of belonging, feel wanted, feel needed, success, sense of achievement, need to compete, need to be seen

- Emotional needs not met: fear, shame, remorse, guilt, anger, frustration, sadness, loss, disappointment, love, support

- Values in conflict: honesty, autonomy, sense of fairness or justice, beneficence, dignity, honor

Labels or Inaccurate Assumptions That Can Block Resolution

- Difficult family

- Passive-aggressive coworker

- Not a team player

- Power-hungry

- Control freak

- Arrogant

- Intellectual snob

- Thinks she is better than anyone else

- Just wants to be the center of attention

- He just wants everyone else to be miserable, too

- Uncooperative

- She just wants to get even

- Doesn't care

- Disengaged

- Lazy

- Incompetent

- Bully

- He is just an angry person

- Irresponsible

- Dangerous

*Each section describes different aspects of assessing a conflict situation. Observed symptoms, underlying issues, and incorrect assumptions can all impact how the problem is defined and the ability of the participants to resolve the true issues. needs and interests and helping people communicate their interests in a supportive environment.

\section{Mediation Techniques}

Mediators use several techniques that are effective at resolving conflicts. Not every situation requires a mediator, and frequently differences can be managed early and directly by those involved in the situation. Mediation techniques can be integrated into clinical care to assist nurses in recognizing issues and addressing the actual needs of patients, families, and coworkers in order to prevent escalation of conflicts, to address underlying motivations, and to save time and resources by solving the correct problem. These techniques can be integrated into routine clinical care situations.

Effective techniques for improving collaboration and resolving conflicts include listening for understanding, reframing, elevating the definition of the problem, and creating clear agreements. Use of these techniques helps to manage conflict by fostering understanding and acceptance, surfacing and acknowledging underlying interests or needs, identifying common ground, and communicating clearly regarding future actions that enable each person to feel that his or her needs have been addressed. Table 3 summarizes these four techniques; exercises for practicing each of these techniques appear below.

\section{The Role of the Advanced Practice Nurse}

The advanced practice nurse (APN) can play a role in creating a healthy work environment by fostering collaborative relationships. ${ }^{2} 9$ Components of the APN's role include educating staff, serving as a role model, developing and working in collaborative and interdependent relationships, negotiating resources, and establishing effective linkages to accomplish goals. ${ }^{29}$ Incorporating mediation techniques into the daily work of the APN allows nursing and physician colleagues to observe effective conflict management strategies, helps meet the needs of patients and families 
TABLE 3 - Mediation Techniques

\begin{tabular}{ll} 
Technique & Description \\
\hline Listening for Understanding & $\begin{array}{l}\text { Listening openly without } \\
\text { interrupting, giving advice, } \\
\text { judging, or asking immediate } \\
\text { questions }\end{array}$
\end{tabular}

Reframing

Elevate the Definition of the Problem

Clear Agreements
A statement or response that acknowledges the emotion, removes the inflammatory language, restates the problem or issue, and seeks clarification or validation from the speaker

A statement or response that combines multiple opposing or disparate positions by reflecting back an issue that is common to everyone involved

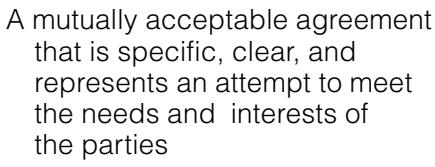

A mutually acceptable agreement that is specific, clear, and represents an attempt to meet the needs and interests of the parties

\section{Purpose}

Allows speaker to feel heard and control content of information; allows listener to hear underlying issues, show empathy, and make open assessments of the situation without jumping to conclusions or confirming assumptions

Through reframing, one can neutralize the language, assess underlying issues, inform the speaker that you understand what they are saying, and redirect the conversation from a confrontational mode into a problemsolving mode

To acknowledge issues that are important to each person while establishing common ground; to demonstrate commonality and encourage each person to work together toward a common goal; to regain trust

Guide future behavior; provide a foundation for trust and a plan for addressing future differences that may arise in a nonadversarial manner; and minimizes the consequences of unresolved conflict within the unit.

\section{Exercises for Learning Mediation Techniques}

The systematic management of conflict makes use of a broad continuum of complementary techniques and processes for addressing differences. Just as clinical care includes alternative treatment modalities, the process of conflict management also has a range of alternatives for preventing, addressing, deescalating, and resolving disputes. Effective conflict management requires a combination of skills and processes for meeting the needs and interests of various stakeholders. Integrating these techniques into the clinical environment can effectively reduce the level of conflict and prevent a unit from becoming dysfunctional. The techniques listed below are but a few of the complementary tools that are useful in managing healthcare conflicts.

\section{Technique 1: Listening for Understanding}

Listening for understanding is more than just receiving information, it is a multilayered technique that entails listening without judgment, without planning a response, and without devising solutions or offering advice. Truly attempting to understand what is occurring for another person requires centering, focus, and presence. Taking in information that includes facts, emotions, body language, and context enables the listener to develop an understanding of the needs, interests, and values of the speaker. Listening at this level enables the listener to fully assess the problem before diagnosing or treating the situation. In a busy critical care environment, there is often a perception that there is insufficient time for this level of interaction. However, this technique can be applied to every interaction without an additional commitment of time. Additionally, achieving an understanding of the underlying issues enables the listener to address the correct problem rather than solve the wrong problem, which results in long-term time sav- 
ings. A method for practicing this technique is described below.

\section{Exercise: Listening for Understanding}

Exercise 1: Pair up with a coworker. One person is the listener and the other the speaker. For 60 seconds, tell the listener about your favorite teacher from school, a preceptor, a mentor, or a family member. The listener should listen for insight into what is important to this person. What made this person choose this story to tell? What do the characteristics of the teacher described in the story tell you about the speaker's interests or values? Validate your observations with the speaker. Now switch roles and repeat the exercise. What level of understanding were you able to gain by listening in this fashion? Was it more time consuming to listen in this way for 60 seconds than it would have been if you were just listening to the facts of the story?

\section{Application to Clinical Practice}

Do this exercise with a new staff nurse. Consider the information that you obtain. Are the needs or interests you identified a match with the type of preceptor with whom he or she has been? Is the way orientation is structured compatible with what matters to this person? For example, a nurse describes a teacher who took the time to encourage her to explore many different ways of solving a particular problem, and she describes the happiness she felt upon being able to problem solve on her own. Is a very structured and compulsive preceptor a good fit for this individual?

Exercise 2: Practice this with a patient's family member who has a concern or with a coworker who has a problem at work: Listen for 90 seconds without interrupting, asking questions, or offering advice. Listen as you did in Exercise 1 for what is important, for what needs or interests you hear identified. Are there points in the story in which the individual seems more upset? What is the person discussing when there is more energy in his or her speech? Are any words or themes being repeated? While you are listening, be aware of yourself. Are you planning a response? Are you preparing mentally with a solution to the problem? Have you decided you know what the problem is before the speaker finishes talk- ing? What does it feel like to listen without the obligation of replying?

\section{Application to Clinical Practice}

Assume that in doing Exercise 2, a patient's family member expressed the following concerns: "No one has come to talk to us today. We don't know what is going on with him. Isn't there a doctor we could talk to? When will be be taken off the ventilator and transferred to the other unit? We thought he would be extubated yesterday and he is still here. He doesn't like the ventilator; can't you do something to make him more comfortable? The nurse last night gave him medication to help him sleep. Why can't you do that? We have been here for a week and there is never the same nurse. Will you be here tomorrow or will there be someone else? We have other family who would like to see him. When will he move out of the ICU?"

What is this family really requesting? What do they need? Would it be difficult to listen to this string of questions without interrupting or becoming frustrated? What would be your response to this family? What sentence could reflect what you hear and summarize what is important for the family member, such as: "It must be frustrating to not have the information you need to make decisions," or "You sound like you are concerned that he is not getting the attention he needs." Would these statements have a different effect than trying to answer each question or giving detailed clinical explanations of why the patient cannot have sedatives during vent weaning?

\section{Technique 2: Reframing}

Listening for understanding provides information as to what is happening for someone and helps to surface the issues. The next step is deciding how to respond to the information. The response should let the speaker know that you have heard what he or she is trying to say. Additionally, if the person is emotionally upset, it is difficult for them to hear any response. Deescalating a person who is upset allows him or her to better hear your comments. Reframing the statements as you respond is one way of surfacing the issues while helping the patient or family member stay engaged and hear your message. Reframing accomplishes many functions. Through 
reframing, it is possible to remove inflammatory language, acknowledge or clarify the underlying issues, inform the speaker that you understand what he or she is saying, and redirect the conversation from a confrontational mode into a problem-solving mode.

Reframing is a technique that requires practice, particularly for those who are uncomfortable when an individual's words feel like an attack or an insult. Mediators frequently use reframing to create a safe space for individuals to have difficult conversations. Reframing strong language while acknowledging the emotion helps people stay in the dialogue. Too frequently, a confrontational statement elicits a "fight or flight" response that can lead to a caustic reply, withdrawal, or walking away. Practicing the technique of reframing allows the others to stay in the conversation in order to work toward solving the problem. Often, the person who is upset will make a strong statement that places blame on someone who is not present. Reframing helps to focus the conversation back on the speaker and away from the person who is not present. This helps to create a space for surfacing the issues and for removing blame from the conversation.

There are four steps in reframing a statement:

- acknowledge the emotion

- remove the inflammatory language

- restate the problem or issue

- request or wait for clarification or validation from the speaker.

Several examples of reframing, and a reframing exercise, are listed below:

\section{Examples of Reframing}

Statement 1: "Doesn't anyone know what they are doing around here?"

Reframed: "You seem to be frustrated, what information would be helpful to you?'

Statement 2: "He is the most incompetent doctor I have ever seen!"

Reframed: "You sound upset. What would help you feel assured that your dad is receiving good care?

\section{Reframing Exercise}

There are many variations in crafting a reframed statement. Reframe the following statements:
Statement 1: "I can see you have to be a friend of who's in charge to get a decent assignment around here!"

Statement 2: "You know, any first-year resident would know the dosing for digoxin; this guy must be some kind of idiot!"

Statement 3: "Could the people in the lab be any more incompetent? They just called and said they can't use the sample I sent down."

\section{Technique 3: Elevating the Definition of the Problem}

Staying engaged in resolving conflict requires that people feel like there is a foundation for collaborating. Often in a conflict it seems as though there is no area of agreement or any desire by the other person or group to address the issues. Finding common ground is a principle of mediation that enables individuals to find a starting point for further agreement. Incrementally building on a series of small agreements is the only means of resolving a complex dispute. When trust has been damaged between the people involved in the conflict, it is necessary to slowly allow them to regain that trust through a series of small commitments. Sometimes agreeing to meet at a certain time is the first point of agreement.

In addition to reestablishing trust, finding common ground also enables people to begin to see the whole person again rather than just a symbol of one's angst. It is sometimes difficult to agree on anything with the person or group who is disrupting your life. Finding common ground is the first step in humanizing the situation. Through agreement with someone, there is recognition of each person's need to be engaged in the process. This mutual recognition of need forms a foundation for collaborating and facilitates the process of reflecting on one's own contribution to the conflict situation.

Finding common ground is the first step toward solving the problem together. One method for finding common ground is through elevation of the problem's definition. Typically, people enter into a dispute with their own definition of what has gone wrong and their own idea of what is needed to fix it. The problem definition frequently involves blaming the other person for what he or she 
has done or not done and the solution is typically a position demanding that something be done about it. This positioning is the result of multiple definitions of the problem and can make for an unproductive conversation in which each person is working toward his or her position as the solution to one's own definition of the problem and no one is working in the same direction. Elevating the definition of the problem is a technique that incorporates statements made by each person into a general definition of something everyone agrees is worth their effort. Once there is agreement on the generalized definition, it is easier to build trust and prevent continued debate over predetermined solutions. With this technique, one can take seemingly opposing views and blend them into a statement that helps everyone begin working in the same direction. Several examples of this technique follow.

\section{Example 1: Elevating the Definition of the Problem}

Nurse: "That doctor does not have adequate clinical training to be doing that procedure."

Physician: "Credentialing of physicians is the job of physicians and not nurses."

Elevated Definition Statement: "Finding a safe and effective way to provide care for this patient is important to all of us."

\section{Example 2: Elevating the Definition of the Problem}

Patient: "That nurse is the worst nurse I have ever seen and can't even start an IV without sticking me three times!"

Orientee: "No matter how hard we try, you keep finding fault with everything we do. Why don't you just go to another hospital?"

Elevated Definition Statement (Preceptor): "You both sound like you care a great deal about ensuring good care and getting the support you both need is important."

\section{Exercise: Elevating the Definition of the Problem}

Practice combining the two statements below into an elevated definition statement that reflects an interest or need that is common to both speakers:

\section{Exercise 1:}

Staff Nurse: I cannot work with an overbearing charge nurse who does not support us when we need help on the floor.

Charge Nurse: You always complain about your assignments and you never ask for help when you need it. I can't read your mind!

\section{Exercise 2:}

Nurse: The lab is always screwing up and losing the specimens and then demanding that we draw another one from patients who are difficult to draw.

Lab Tech: If they would just pay attention and label the specimens correctly, we would not have to reject them and request an additional sample.

\section{Technique 4: Making Clear Agreements}

The goal of managing conflict is to develop clear agreements that guide future interactions. Differentiating between a discussion and decision is essential. The fourth technique used by mediators is making clear agreements. Often a conversation is simply an exchange of information or opinions and no plan is made to follow up on the problems identified. Alternatively, there may be a plan, but each person leaves the conversation with a different perception of the agreement. Making clear agreements helps manage expectations and guide future behavior. Using good communication, understanding the needs of others, following through on promises, using persuasion and not coercion, and accepting the perceptions and ideas of others can result in clear agreements that prevent future conflict and provide a framework for dealing with differences.

\section{Components of a Clear Agreement}

Specificity: Determine who will do what and when.

Clarity: Terms are easily understood; define terms as necessary.

Mutuality: Be sure everyone has taken responsibility and has something they have promised to do.

Terms are actually agreed to and agreement is sought directly: "Is this something you feel comfortable with and able to do?"

Follow-up: Address what happens if a conflict situation arises again or others be- 
come involved who are not aware of the agreement.

Include a plan for what will occur in the event someone does not adhere to the agreement. Determine what will be the consequences or alternative actions taken.

\section{Sample Agreement}

\section{Communication Plan with Family of Patient A}

The ICU staff and Mr. A.'s family agree that clear and consistent communication is necessary for good working relations and for the promotion of quality care of $\mathrm{Mr}$. A. Mr. B ( Mr. A.'s brother) agrees that he will serve as the family spokesperson and will meet twice each day (9:00 AM and 8:00 PM) with the ICU charge nurse for an update on Mr. A.'s condition.

The ICU staff agrees to keep the ICU charge nurse updated and will page the physician when Mr. B. arrives on the unit. The ICU staff agree to contact Mr. B. by phone should any serious changes occur in Mr. A's condition.

Mr. B. agrees to inform all family members to refrain from calling the ICU with questions without talking to Mr. B first.

In the event there is a disagreement between Mr. B. and any of the clinical staff, Mr. B. and the staff agree to seek the assistance of the hospital mediator to discuss further issues and that all discussions will be held in the family conference room.

Signed: (Mr. B)

\section{Date:}

Signed: (ICU Manager)

Date:

\section{Application of Mediation Techniques: A Clinical Conversation}

The following dialogue, based on an actual scenario, demonstrates the application of the techniques described in the article to a common clinical situation. Estimate the time invested in the conversation using the mediation techniques as compared to the time that would be invested in managing unresolved or unacknowledged issues if the techniques were not applied.
Scenario: The patient is a 58 -yr-old male with end-stage liver disease, on continuous veno-venous hemofiltration and who is not presently a candidate for transplant. He has two children from his first marriage who have not had much contact with him. His current wife is distraught and there has just been an explosive argument between her and the patient's son at the bedside. The son has left the unit claiming he is going to have her declared incompetent and have security remove her from the hospital. The nurse assigned to the patient has just stepped in to address the conflict.

Dialogue (techniques are noted in italics):

Wife: That son of his has no right to show up now expecting to be in charge of what happens to my husband when his son has been no where to be seen for the past 9 years! I do not want him allowed in here making things worse, upsetting me and my husband! I will get a restraining order against him if you can't keep him out!

Nurse: I'm sorry that you are going through all of this, it is clearly upsetting to have one more thing to have to deal with. (Listening for understanding)

Wife: Upsetting? It is more than upsetting! I am angry! I have been by my husband's side through this whole illness. It is not fair that something like this should happen to him. It's not like he drinks or takes drugs or did anything to deserve this. This past year has been horrendous and you would think someone would have offered to help us. I have managed everything on my own and I don't need their help now. Why can't he have any water? His mouth is so dry he is developing sores and the nurse last night didn't even give him a bath.

Nurse: I did not mean to minimize what you have been through, anyone in your situation would be angry. Its not fair that good people have bad things happen to them. (Acknowledge emotion and sense of justice, normalize her feelings, refocus the conversation on the wife, NOT the patient's son-reframing)

It sounds like you have been very loyal and he has had to rely on you a lot during his illness. (Acknowledge ber need for recognition, value of loyalty)

You obviously care a great deal about what happens to him and providing good 
care is our goal too. (Identify the common ground; elevate the definition of the problem)

What do you need that would be helpful in managing this situation? (Listen for understanding/surface the issues)

(Note: The nurse did not try to justify why a bath was not given or reasons for water restriction; she did not become defensive about allegations of bad care. These statements were ways the wife was trying to express her unmet needs, not the needs of the patient.)

Wife: I need to know that someone cares about him and that no one is going to harm him. He is so vulnerable and if I am not watching over him every minute, I am afraid of what will happen to him.

Nurse: It sounds like this is a pretty scary situation for you. What are you afraid will happen? (Surface the unmet emotional need - fear, listening for understanding)

Wife: I am afraid he will die. (Begins crying... Emotion is expressed)

Nurse: Why don't I take you to the private family room for a little while, you can return whenever you are ready. Is there someone you would like me to call or would you like some time to talk with the social worker? She can help you identify several resources that may be helpful for you. Is that something you would like to do? (Options generated, seeking clear agreement)

Wife: Yes. Thank you for understanding.

(Note: The conversation did not revolve around the visitation policy, the threat of a restraining order, the actions of the son who is not present for the discussion, or the allegations of poor care provided by the other nursing staff. The nurse listened, acknowledged potential unmet needs, clarified the needs, reframed the angry statements, and elevated the definition of the problem. The nurse and the patient's wife can now discuss what will meet her needs. Once the wife feels her fears have been addressed, she will be able to talk more clearly about what steps to take in dealing with the son and his need to see his father. In real time, this conversation takes 2 minutes and effectively addresses the real issues driving the confrontation. The conflict is deescalated, the wife's needs are met, and the nurse is free to continue caring for the patient.)

\section{$\square$ Conclusion}

Managing conflict to create healthy work environments is an essential component in the evolution of the delivery of critical care services. Collaborative working relationships are important in establishing these environments, in ensuring safe patient care, and in decreasing costs associated with unresolved conflicts. Integrating the techniques used by mediators of listening for understanding, reframing, elevating the definition of the problem, and creating clear agreements can foster collaboration. Better working relationships can improve patient outcomes, improve retention and recruitment of staff, and improve patient satisfaction.

\section{References}

1. Baker C, Belinger J, King S, Salyards M, Thompon A. Transforming negative work cultures: A practical strategy. J Nurs Admin. 2000;30(7/8):357-363.

2. The National Academy of Sciences. Keeping Patients Safe: Transforming the Work Environment of Nurses. 2004. Full report including Appendix B-Interdisciplinary Collaboration, Team Functioning, and $\mathrm{Pa}-$ tient Safety; 2004.

3. Racy MF, Ceronsky C. Creating a collaborative environment to care for complex patients and families. AACN Clin Issues. 2001;12(3):383-400.

4. Press Ganey Satisfaction Report, Vol. VII. August 2003. Available at: http://www.jcaho. org/accredited+organizations/ambulatory+ care/sentinel+events/sentinel+event+statistics. htm. Accessed March 29, 2004.

5. Forte P. The high cost of conflict. Nurs Econ. 1997;15(3):119-125.

6. Slaikeu K, Hasson R. Controlling the Costs of Conflict: How to Design a System for Your Organization. Jossey-Bass; 1998.

7. AHA and CPR Institute for Dispute Resolution. Managing Conflict in Health Care Organizations - A Handbook. AHA and CPR Institute for Dispute Resolution; 1995.

8. Henneman E, Dracup K, Ganz T, Molayeme O, Cooper C. Effect of collaborative weaning plan on patient outcome in the critical care setting. Crit Care Med. 2001;29(2):297-303.

9. Joint Commission on Accreditation of Healthcare Organizations. JCAHO Root Causes of Sentinel Events-1995-2002. 
Available at: http://jcaho.com/accreditedor ganizations/ambulatorycare/sentinelevents.

10. Grap M, Strickland D, Tormey L, et al. Collaborative practice: Development, implementation and evaluation of a weaning protocol for patients receiving mechanical ventilation. Am J of Critical Care. 2003; 12(5):454-460.

11. Chassin M, Becher E. The wrong patient, quality grand rounds, Ann Intern Med. 2002; 136(11):826-833.

12. Pizzi L, Goldfarb N, Nash D. Evidence Report/Technology Assessment, No. 43. Making Health Care Safer: A Critical Analysis of Patient Safety Practices. AHRQ Report, Prepared by UCSF and Stanford University Evidence-based Practice Center, chp. 40 (Promoting a Culture of Safety). Available at: http://www.ahcpr.gov/clinic/ptsafety/ chap40.htm. Accessed March 29, 2004.

13. Ang M. Advanced communication skills: Conflict management and persuasion. Acad Med. 2002;77:1166.

14. Klein E, Jackson J, Kratz L, et al. Teaching professionalism to residents. Acad Med. 2003;78(1):26-34.

15. Hawryluck L, Espin S, Garwood K, Evans C, Lingard L. Pulling together and pushing apart: Tides of tension in the ICU ream. Acad Med. 2002;77(10):S73-S76.

16. Kritek P. Negotiating at an Uneven TableDeveloping Moral Courage in Resolving our Conflicts, 2nd ed. New York, NY: John Wiley and Sons; 2002.

17. Van Ess Coeling H, Cukr PL. Communication styles that promote perceptions of collaboration, quality and nurse satisfaction. $J$ Nurs Care Qual. 2000;14(2):63-74.

18. Hawk C, Buckwalter K, Byrd L, Cigelman S, Dorfman L, Ferguson K. Health professions students' perceptions of interprofessional relationships. Acad Med. 2002;77(4): 354-357.

19. Cloke K, Goldsmith J. The End of Management and the Rise of Organizational Democracy. San Francisco, Ca: JosseyBass; 2002.

20. Valentine P. Management of conflict: Do nurses/women handle it differently? J $A d v$ Nurs. 1995;22(1):142-149.

21. Anderson R, McDaniel Jr R. Managing health care organizations: Where professionalism meets complexity science. Health Care Manage Rev. 2000;25(1):8392.

22. Schein E. The Corporate Culture Survival Guide. San Francisco, Ca: Jossey-Bass; 1999.

23. Marcus L, Dorn B, Kriteck P, Miller V, Wyatt J. Renegotiating Health Care: Resolving
Conflict to Build Collaboration. San Francisco, Ca: Jossey Bass; 1995.

24. Fisher R, Brown S. Getting Together: Building Relationships as We Negotiate. New York: Penguin Books; 1998.

25. Beaudoin L, Edgar L. Hassles: Their importance to nurses' quality of work life. Nurs Econ. 2003;21(3):106-113.

26. Pfifferling JH, Developing and implementing a policy to deal with disruptive staff members. Oncology Issues. 2003;18(3):16-21.

27. Moore C. The Mediation Process: Practical Strategies for Resolving Conflict, 3rd ed. New York, NY: John Wiley \& Sons; 2003.

28. Suchman A, The influence of health care organizations on well-being. West J Med. 2001;174(1):43-47.

29. Disch J, Walton M, Barnsteiner J. the role of the clinical nurse specialist in creating a healthy work environment. AACN Clin Issues. 2001;12(3):345-355.

\section{Additional Resources}

\section{Books}

Cloke K, Goldsmith J. Resolving Conflicts at Work. San Francisco, Calif: Jossey-Bass; 2000.

Cloke K, Goldsmith J. The Art of Waking People Up. San Francisco, Calif: Jossey-Bass; 2003.

Constantino CA, Merchant CS. Designing Conflict Management Systems: A Guide to Creating Productive and Healthy Organizations. San Francisco, Calif: Jossey-Bass; 1996.

Crum T. The Magic of Conflict. New York, NY: Simon and Schuster; 1987.

Dubler N, Marcus L. Mediating Bioethical Disputes: A Practical Guide. United Hospital Fund of New York; 1994.

Fisher R, Ury, W. Getting to Yes: Negotiating Agreement Without Giving In. New York, NY: Penguin Books; 1991.

Isaacs W. Dialogue and the Art of Thinking Together. New York, NY: Doubleday; 1999.

Kolb D, Williams J. The Shadow Negotiation. New York, NY: Simon \& Shuster; 2000.

Madonik B. I Hear What You Say, but What Are You Telling Me. The Strategic Use of Nonverbal Communication in Mediation. New York, NY: John Wiley and Sons; 2001.

Patterson K, Grenny J, McMillan R, Switzler A. Crucial Conversations. Tools for Talking When Stakes are High. New York, NY: McGraw Hill; 2002.

Wheatley M. Turning to One Another- Simple Conversations to Restore Hope to the Future. San Francisco, Calif: Berrett- Koehler Publishers; 2002. 


\section{Web Sites}

American Health Lawyers Association: www. healthlawyers.org

Articles/trainings/service-provider listings: www. mediate.com/healthcare

Association for Conflict Resolution: www. acresolution.org (www.acrnet.org)

Conflict Resolution Institute: www.crinfo.org

CPR Institute for Dispute Resolution: www. cpradr.org

Federal Mediation and Conciliation Service: www.fmcs.gov
Health Care Mediations, Inc: www.healthcare mediations.com Indiana Conflict Resolution Institute Database: www.spea.indiana.edu/icri/datalist.htm

Mediate Calm, Inc: www.mediatecalm.ca

Program for Healthcare Negotiation and Conflict Resolution-Harvard School of Public Health: www.hsph.harvard.edu/php/phencr

Program on Negotiation at Harvard Law School: www.pon.harvard.edu 